\section{Response to Comments on "Impacts of Biodiversity Loss on Ocean Ecosystem Services"}

Boris Worm, ${ }^{1 *}$ Edward B. Barbier, ${ }^{2}$ Nicola Beaumont, ${ }^{3}{ }^{3}$. Emmett Duffy, ${ }^{4}$ Carl Folke, ${ }^{5,6}$
Benjamin S. Halpern, ${ }^{7}$ Jeremy B. C. Jackson, ${ }^{8,9}$ Heike K. Lotze, ${ }^{1}$ Fiorenza Micheli, ${ }^{10}$ Stephen R. Palumbi, ${ }^{10}$ Enric Sala, ${ }^{8}$ Kimberley A. Selkoe, ${ }^{7}$ John J. Stachowicz, ${ }^{11}$ Reg Watson $^{12}$

We show that globally declining fisheries catch trends cannot be explained by random processes and are consistent with declining stock abundance trends. Future projections are inherently uncertain but may provide a benchmark against which to assess the effectiveness of conservation measures. Marine reserves and fisheries closures are among those measures and can be equally effective in tropical and temperate areas-but must be combined with catch-, effort-, and gear restrictions to meet global conservation objectives.

$\mathrm{W}$

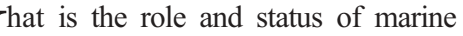
biodiversity in sustaining ocean ecosystem services such as food supply, water quality control, and ecosystem stability? In our recent study, we addressed this question using meta-analysis of published experimental data, historical time series, global catch trends, and studies of marine reserves and fisheries closures (1). We found that in all of these independent data sets, biodiversity was positively related

${ }^{1}$ Department of Biology, Dalhousie University, Halifax, NS, Canada B3H 4]1. 'Department of Economics and Finance, University of Wyoming, Laramie, WY 82071, USA. ${ }^{3}$ Plymouth Marine Laboratory, Plymouth PL1 3DH, UK. ${ }^{4}$ Virginia Institute of Marine Science, Gloucester Point, VA 23062-1346, USA ${ }^{5}$ Department of Systems Ecology, Stockholm University, Stockholm SE-106 91, Sweden. ${ }^{6}$ Beijer International Institute of Ecological Economics, Royal Swedish Academy of Sciences, SE-104 05, Stockholm, Sweden. ${ }^{7}$ National Center for Ecological Analysis and Synthesis, Santa Barbara, CA 93101, USA. ${ }^{8}$ Center for Marine Biodiversity and Conservation, Scripps Institution of Oceanography, La Jolla, CA 920930202, USA. ${ }^{9}$ Smithsonian Tropical Research Institute, Box 2072 Balboa, Republic of Panama. ${ }^{10}$ Hopkins Marine Station, Stanford University, Pacific Grove, CA 93950, USA. ${ }^{11}$ Section of Evolution and Ecology, University of California, Davis, CA 95616, USA. ${ }^{12}$ Fisheries Centre, University of British Columbia, Vancouver, BC, Canada V6T $1 Z 4$.

*To whom correspondence should be addressed. E-mail: bworm@dal.ca to productivity, stability, and the supply of ecosystem services. The comments by Wilberg and Miller (2), Jaenike (3), and Hölker et al. (4) focus almost exclusively on our usage of catch trends and our projection of a possible fisheries collapse after accelerated biodiversity loss.

First, Wilberg and Miller (2) argue that under certain assumptions, a random process may generate declining trajectories in the global catch data that are similar to observed trends. They hypothesize that the increasing proportion of time series that fall below $10 \%$ of the maximum catch (our operational definition of collapse) could be a simple, accumulating function of time. If this were the case, recovery after a collapse would certainly occur. Yet in reality, recoveries of collapsed stocks are often rare, as discussed below. Furthermore, their supporting formula assumes an independent, identically distributed (iid) times series. However, the assumption of independence among data points does not hold for the autocorrelated random series that they use in their simulations, nor for the catch data that we used in (1).

Despite these shortcomings, we were able to test Wilberg and Miller's hypothesis that increasing length of time series is correlated with increasing likelihood of collapse across large marine ecosystems (LMEs). We used all avail- able fisheries catch data from the catch database of the United Nations Food and Agriculture Organization (FAO) and other sources, as outlined in (1). Results show that for the 64 LMEs included in our original study, there is no relation between the average start year of a fishery and the likelihood of a collapse (Fig. 1A). This means that ecosystems that have been fished for longer do not necessarily show more frequent collapses. Furthermore, there is an inverse correlation between the average lifetime of a fishery (the length of time over which it produced catches) and the proportion of fisheries that are collapsed (Fig. 1B). This refutes the idea that longer catch series are more prone to collapse than shorter ones, as suggested in (2).

Wilberg and Miller further note that the causes for a $90 \%$ decline in catch could be complex and do not necessarily indicate overexploitation (2). We agree, and emphasize that the purpose of our study was not to test hypotheses about the putative causes of declining fisheries but to elucidate the role of marine biodiversity for stabilizing fisheries catches and other ecosystem goods and services. Although there may be disputes about driving causes in individual cases, for global fisheries as a whole there is no reasonable doubt that most major declines in stock biomass and in corresponding yields are due to unsustainable levels of fishing pressure (5-8). Global assessments of stock biomass (7) support our conclusions that a large fraction of the world's fished biodiversity is overexploited or depleted (24\% of assessed stocks in 2003), that this fraction is increasing (from $10 \%$ in 1974), and that recovery of depleted stocks under intense management is still an exception (1\% in 2003). An in-depth analysis of fish stock collapse and recovery using available abundance data (not catches) also concluded that there is little evidence for rapid recovery from prolonged declines (9). This undermines Wilberg and Miller's argument of a random process, because population recovery should be just as likely as collapse under their assumption.

In a second comment, Jaenike (3) focuses on the statistical model used to approximate time series trends from global catch data. He points out the clustering of points above and below the
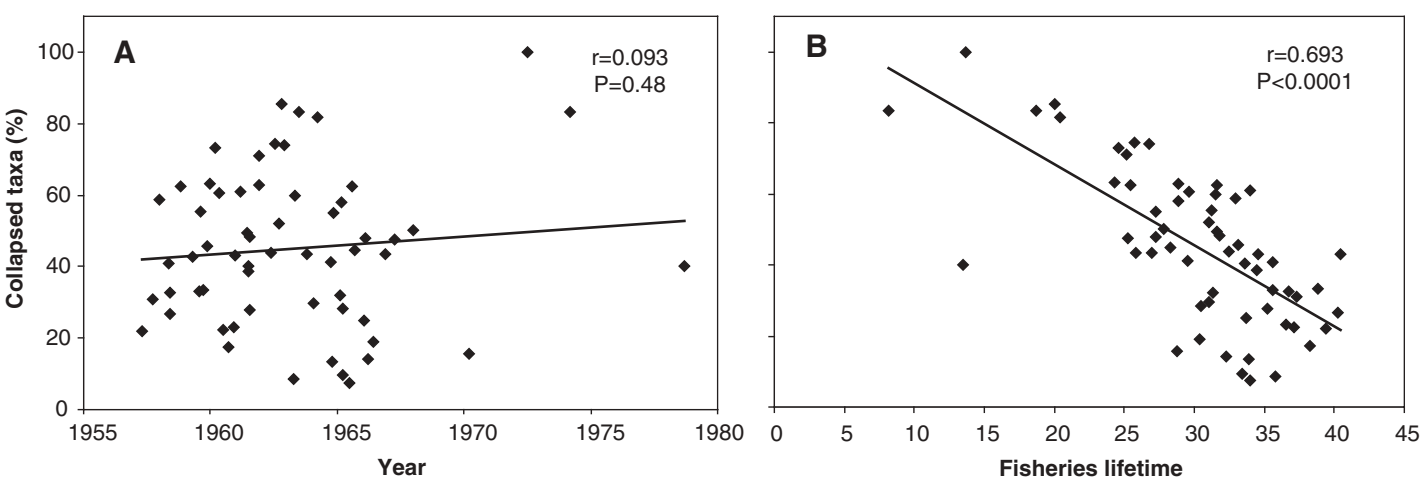

Fig. 1. Fisheries collapses in LMEs as a function of time. Percentage of taxa that are currently producing less than $10 \%$ of the maximum catch ("collapsed taxa"), as a function of $(\mathbf{A})$ average start year of the fishery, and (B) average lifetime of a fishery. 
Table 1. Meta-analysis of tropical versus temperate protected areas. Log response ratios $(\ln R)$ were calculated for variables with three or more study sites $(N)$ in each category. $\mathrm{Cl}$, confidence interval.

\begin{tabular}{|c|c|c|c|c|c|c|}
\hline \multirow{2}{*}{ Response variable } & \multicolumn{3}{|c|}{ Tropical areas } & \multicolumn{3}{|c|}{ Temperate areas } \\
\hline & $N$ & $\ln R$ & $95 \% \mathrm{Cl}$ & $N$ & $\ln R$ & $95 \% \mathrm{Cl}$ \\
\hline Species richness & 25 & 0.090 & -0.08 to 0.26 & 18 & 0.250 & 0.15 to 0.34 \\
\hline Fishable species & 19 & 0.040 & -0.06 to 0.15 & 12 & 0.050 & -0.12 to 0.22 \\
\hline Catch per unit effort & 6 & 0.500 & 0.25 to 0.76 & 3 & 1.120 & 0.36 to 1.88 \\
\hline Variability & 8 & -0.330 & -0.62 to -0.03 & 14 & -0.110 & -0.35 to 0.13 \\
\hline
\end{tabular}

regression line that we have fitted to the time series [figure $3 \mathrm{~A}$ in (1)]. We note that this is a normal feature of any autocorrelated time series (10). Jaenike then applies an ad hoc piecewise regression model to the latter part of the catch series (1967 to 2003) and shows that a different projection ensues if that model is used to extrapolate into the future. Breaking the time series at 1967 would only be justified if a different process were driving the latter part of the time series. This assumption is not backed by any mechanism, such as a change in management at that time. We agree with his general point, though, that not too much reliance should be given to any one model and that alternative models are possible (for example, a logistic regression would be an obvious choice). Likewise, future projections from such alternative models would yield different results but would involve equally strong assumptions about the dynamics of global fisheries. This, however, was not the point of our study. Rather, we sought to highlight that there is a progressive and consistent loss of the biodiversity that fuels our fisheries. This trend is negative and increasing, no matter which model is applied to approximate it. It is seen in all the available data, including the FAO report on the status of world fisheries, which is based on stock assessments rather than catch trends (7). We therefore stand by our conclusion that the erosion of marine biodiversity is threatening world seafood supplies and emphasize that the protection and restoration of biodiversity must be a cornerstone of any rational management regime.

In a third comment, Hölker et al. (4) state that, with respect to future projection of catch trends, it is generally problematic to extrapolate outside the range of available data. Although we agree in principle, a strict adherence to this rule would preclude any use of trend analysis to explore future scenarios. Our study (1) drew attention to the fact that fisheries depend on a limited number of available taxa and that there was a monotonic trend of declining catches affecting an increasing number of taxa since 1950 . We believe that it is appropriate to ask how long this trend could continue before reaching a global limit. This does not amount to a prediction of what is going to happen, nor to an analysis of underlying mechanisms, but it does raise legitimate concerns about the direction of the trend. This reasoning is well accepted in population viability analysis, for example, where projections can span several hundred years (11). We acknowledge that such projections are generally uncertain and may be used best as scenarios, hypotheses, or benchmarks against which to measure future progress in fisheries management and biodiversity conservation.

Such progress will rely on the broad implementation of effective conservation measures. Marine reserves and fisheries closures have received much recent attention because they can generate rapid conservation and fisheries benefits $(12,13)$. We showed that such benefits typically include an increase in species diversity and a restoration of services associated with the productivity and stability of the ecosystem (1). Hölker et al. question the generality of that analysis. They suggest that our database was biased toward tropical areas; we note, however, that temperate reserves constituted $40 \%$ of the available data. They further ask whether reserves or closures would enhance biodiversity and ecosystem services equally in tropical and temperate areas, and whether they could benefit groundfish or pelagic fisheries. We used meta-analysis of our published database (1) to compare responses to protection between temperate and tropical areas (Table 1). Responses were similar in direction, but diversity and catch per unit effort increased more in temperate areas, whereas temporal variability was reduced more in tropical areas. We also note that several prominent examples of groundfish recovery were associated with temperate closures (14-16). These results suggest that marine reserves and fisheries closures in temperate areas result in increased fish abundance and the recovery of biodiversity, similar to what has been observed in the tropics. Unfortunately, we cannot presently assess the specific effects of closures on pelagic fisheries because of a lack of published data. Simulations indicate that largescale movements of target species and their fisheries may complicate both the siting and the assessment of marine reserves aimed at restoring pelagic biodiversity (17-19). We emphasize that closures and reserves are not a panacea and need to be augmented by other measures to reduce total fishing mortality and rebuild depleted marine populations (8). This is particularly true for highly mobile pelagic species. Designing cost-effective instruments to limit catch, fishing effort, and the use of unselective fishing gear are crucial steps to ensure the sustainability of fisheries that depend on these species $(8,20)$.

We conclude that our discussion of a possible seafood collapse has focused much attention on patterns and trends in global fisheries. Although future projections of such trends can be informative benchmarks, they do not represent necessary outcomes. We have attempted to refocus attention on what is known now. The ongoing erosion of marine biodiversity is exceedingly well documented, as are some of the functional and societal consequences (e.g., 1, 8, 9, 19). There is also a clear understanding of what past mistakes we need to avoid and what we can learn from recent successes, (e.g., 5, 6, 8, 12). Whether ocean ecosystems continue on a trajectory of decline, whether they stabilize, or whether they recover on a large scale will thus depend on our collective societal choices.

\section{References and Notes}

1. B. Worm et al., Science 314, 787 (2006).

2. M. J. Wilberg, T. J. Miller, Science 316, 1285 (2007); www.sciencemag.org/cgi/content/full/316/5829/1285b.

3. J. Jaenike, Science 316, 1285 (2007); www.sciencemag.org/cgi/content/full/316/5829/1285a.

4. F. Hölker et al., Science 316, 1285 (2007); www. sciencemag.org/cgi/content/full/316/5829/1285c.

5. D. Ludwig, R. Hilborn, C. Walters, Science 260, 17 (1993).

6. R. Hilborn et al., Annu. Rev. Environ. Res. 28, 359 (2003).

7. FAO, The State of World Fisheries and Aquaculture 2004 (FAO, Rome, 2005)

8. D. Pauly et al., Nature 418, 689 (2002).

9. J. A. Hutchings, Nature 406, 882 (2000).

10. W. Fuller, Introduction to Statistical Time Series (Wiley, New York, 2nd ed., 1996).

11. P. Armbruster, R. Lande, Conserv. Biol. 7, 602 (1993).

12. F. Gell, C. M. Roberts, Trends Ecol. Evol. 18, 448 (2003).

13. L. D. Rodwell, E. B. Barbier, C. M. Roberts, T. R. McClanahan, Can. J. Fish. Aquat. Sci. 60, 171 (2003).

14. S. A. Murawski, R. Brown, H.-L. Lai, P. J. Rago, L. Hendrickson, Bull. Mar. Sci. 66, 775 (2000).

15. J. A. D. Fisher, K. T. Frank, Mar. Ecol. Prog. Ser. 240, 249 (2002).

16. C. Pipitone, F. Badalamenti, G. D'Anna, B. Patti, Fish. Res. 48, 23 (2000).

17. B. Worm, H. K. Lotze, R. A. Myers, Proc. Natl. Acad. Sci. U.S.A. 100, 9884 (2003).

18. K. D. Hyrenbach, K. A. Forney, P. K. Dayton, Aquat. Conserv. Mar. Freshwat. Ecosyst. 10, 437 (2000).

19. J. K. Baum et al., Science 299, 389 (2003).

20. J. Sibert, J. Hampton, P. Kleiber, M. Maunder, Science 314,1773 (2006)

21. We gratefully acknowledge helpful comments from C. Field and W. Blanchard.

19 December 2006; accepted 2 May 2007 10.1126/science. 1138466 